\title{
Oxidative stress - assassin behind the ischemic stroke
}

\author{
Hanumanthappa Pradeep, Joseph B. Diya, Shivaiah Shashikumar, Golgodu K. Rajanikant \\ School of Biotechnology, National Institute of Technology Calicut, Kerala, India
}

\begin{abstract}
Ischemic stroke is the second leading cause of death and disability worldwide and is associated with significant clinical and socioeconomic implications, emphasizing the need for effective therapies. Several neuroprotective strategies have failed in clinical trials because of poor knowledge of the molecular processes flanked with ischemic stroke. Therefore, uncovering the molecular processes involved in ischemic brain injury is of critical importance. Therapeutic strategies for ischemic stroke remain ineffective, though rapid advances occur in understanding the pathophysiology of the disease. The oxidative stress is one such high-potential phenomenon, the precise role of which needs to be understood during ischemic events. Nevertheless, the studies carried out in preclinical models of ischemic stroke have pointed to the major role of oxidative stress in exacerbating the ischemic injury. Oxidative stress leading to cell death requires generation of free radicals through multiple mechanisms, such as respiratory inhibition, $\mathrm{Ca}^{2+}$ imbalance, excitotoxicity, reperfusion injury and inflammation. Free radicals are highly reactive to all the molecular targets: lipids, proteins and nucleic acids, modifying their chemical structure and generating oxidation-derived products. This review discusses molecular aspects of oxidative stress in ischemic stroke and catastrophes that set up as an aftermath of the trauma.
\end{abstract}

Key words: ischemic stroke, oxidative stress, excitotoxicity, inflammation, apoptosis.

\section{Introduction}

Ischemic stroke constitutes one of the most urgent public health challenges that are threatening the world in the 215t century. Ischemic stroke is the most prevalent type of stroke, constituting about $80 \%$ of all cases of cerebrovascular injury and the leading cause of long-term disability on the global level $[10,92]$. According to the reports, there were 15.3 million incidences of stroke in 2002 alone and the number is projected to increase in the future, if rigorous measures are not adopted to tackle the challenges posed by this disorder [86]. One of the biggest challenges of post-stroke therapy is the short window of treatment, which often makes it impossible for the patient to receive com- petent medical attention in time to minimize devastating and often irreversible brain damage. Ischemic stroke is caused by a blood clot or occluding arteries in the brain which reduces oxygen supply to the critical tissues of the brain. Obstruction of a blood vessel in the brain brings on the rapid cell death in the core of the offended region and actuates damaging mechanisms in the circumferential area; the penumbra. These mechanisms contribute to the generation of factors that initiate cell death. In particular, a complicated series of biochemical cascades take place in the neurons leading to serious damage of the brain tissue $[23,40]$.

Oxidative stress is a disturbance in the oxidantantioxidant balance leading to the potential cellular 
damage. As the brain consumes about $20 \%$ of the body's total oxygen, neuronal cells are more vulnerable than other cells to oxidative damage, although brain constitutes less than $2 \%$ of the total body weight [16]. The significance of oxidative damage as a component of many disease processes in the central nervous system (CNS) is being increasingly recognized. One of the major contributors of oxidative stress is free radicals; superoxide anion $\left(\mathrm{O}_{2} \bullet-\right)$, hydroxyl radical $(\mathrm{HO} \bullet)$, lipid radicals (ROO-) and reactive nitrogen species (RNS) such as nitric oxide (NO) [87]. ROS can alter gene/protein expression by acting as second messenger molecules that can influence intercellular signalling cascades, ultimately affecting cell phenotype and function. Furthermore, they can directly oxidize cellular components leading to impairment of the cell and contributing to disease progression [57].

Experimental stroke studies have confirmed that oxidative stress plays an important role in cerebral damage following ischemia. Oxidative stress is the overproduction of reactive oxygen species (ROS) and other free radicals beyond the ability of the biological sys- tem to neutralize their adverse effects [58]. Radicals are produced at low levels in the cell via many biochemical processes such as the mitochondrial electron transport chain. ROS perform many vital cellular functions including redox signalling and defence against invading bacteria and other microorganisms. When produced in excess quantities, free radicals can injure cells by attacking vital cellular constituents, such as proteins, fatty acids/lipids and DNA. The lethality of ROS can be further augmented by NO to produce reactive nitrogen species such as peroxynitrite (ONOO-), a molecule that causes oxidation and nitration of tyrosine residues on proteins. The current review attempts to explore the major role of oxidative stress in elevating the fatality of the stroke; precisely we are giving the bird view of the free radical generation, resultant oxidative stress and its role in the pathogenesis of stroke (Fig. 1).

\section{Oxidative stress in ischemic stroke}

Cerebral ischemia is the shortage of blood and bloodborne factors to areas of tissues in the brain resulting in cerebral infarction. The main cause of an ischemic

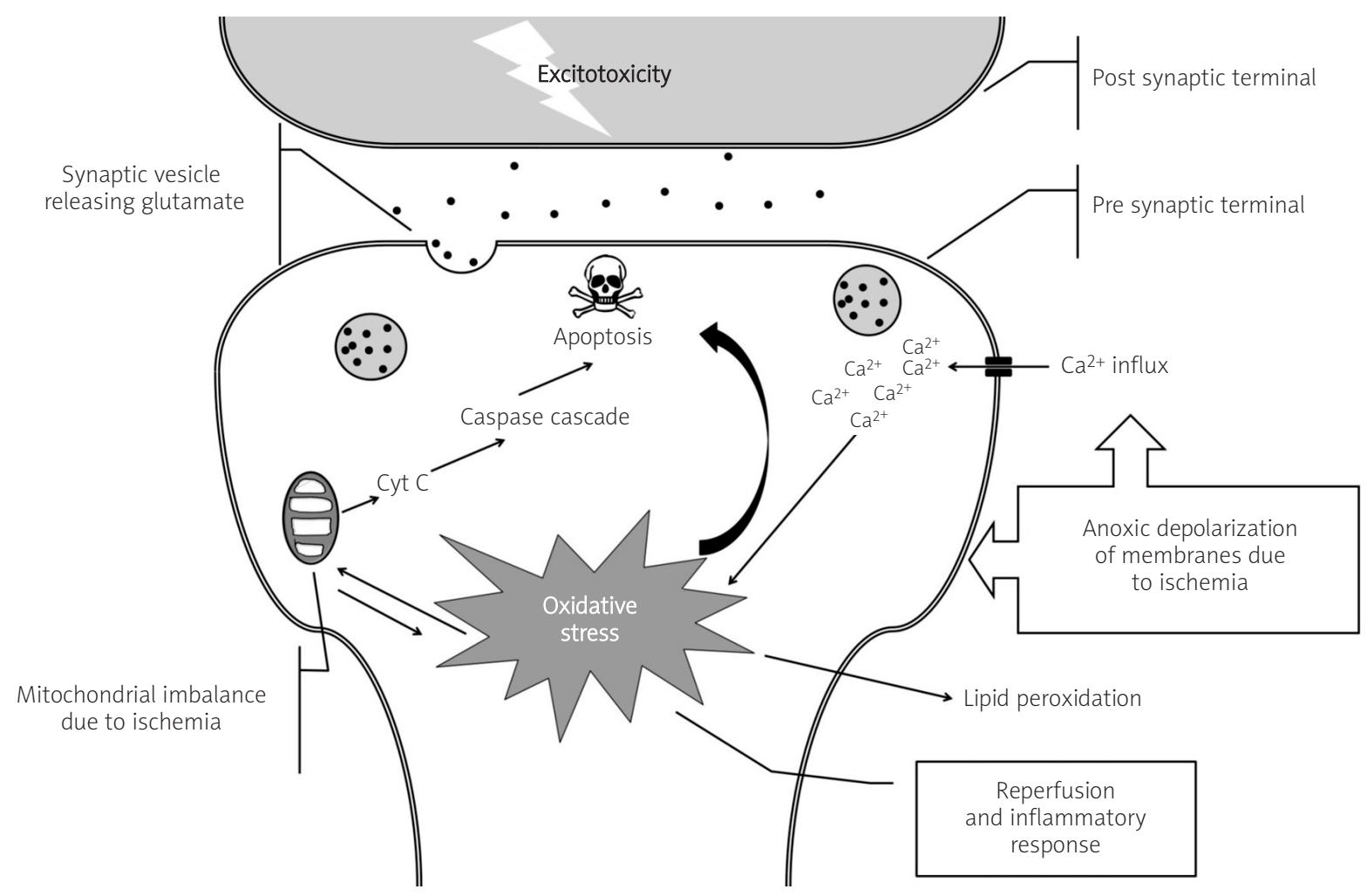

Fig. 1. Potential roles of oxidative stress in an ischemic event. 
stroke is the sudden occlusion of arteries in the brain due to the formation of plaques or clots [55]. Cerebral thrombosis is the most common type of stroke. Build-up of plaques on arterial walls due to atherosclerosis leads to the formation of a thrombus in arteries such as the carotid, resulting in heightened ischemia and cerebral infarction [77]. In some cases, embolization of the thrombus takes place resulting in a wandering clot that can lodge in arteries supplying blood to the brain, ultimately causing an occlusion [93]. Blockage of arteries eventually causes reduction in the perfusion to the brain, setting up an anoxic condition. Consequence of this anoxic state is the decline in the production of high-energy phosphates [97]. Membrane depolarization occurs as an aftermath of severe alteration in the energy homeostasis further triggering eruptive release of excitatory amino acids such as glutamate into the extracellular space [14]. Glutamate induces increased calcium overload in the neurons by acting upon other different receptors. The heightened calcium level is a path for several catastrophes which are crucial for neural cell death [43].

Heightened intracellular $\mathrm{Ca}^{2+}, \mathrm{Na}^{+}$and ADP cause mitochondria to produce destructive ROS. As the brain possesses low levels of antioxidants, neurons are relatively vulnerable to oxidative stress than any other cells of the body [21]. During ischemia, nitric oxide synthase (NOS) activity increases by many folds in the neurons. The glutamate effluxing at synapses, activation of NMDA receptors and elevation in the intracellular $\mathrm{Ca}^{2+}$ level are the main rationalities behind the elevated activity of NOS. In the context of brain ischemia, the activity of NOS is broadly deleterious and their inhibition or inactivation is neuroprotective. In addition, ischemia leads to the generation of superoxide, through the action of NADPH oxide synthases (NOX), xanthine oxidase (XO), cyclo-oxygenase (COX), and leakage from the mitochondrial electron transport chain and other mechanisms $[18,39,73]$. Highly reactive $\mathrm{NO}$ and superoxide are themselves deleterious, however they can combine to form a highly toxic anion, peroxynitrite. The peroxynitrite and other ROS destroy the DNA, actuating the poly(ADP-ribose) polymerase-1 (PARP-1), a DNA repair enzyme. PARP-1 catalyzes the conversion of $\beta$-nicotinamide adenine dinucleotide (NAD+) into nicotinamide (NA) and long polymers of poly(ADP-ribose). As a consequence of PARP-1 over-activation, cellular levels of $\mathrm{NAD}^{+}$depletes impairing $\mathrm{NAD}^{+}$dependent processes such as glycolysis and mitochondrial respiration, which leads to ATP starvation, energy failure and consequently neuronal death [4] (Fig. 2).
Alternatively, non-selective cation channels such as TRPM 2 and TRPM7 can be activated to ensure sustained calcium overload and death [21]. As the extra cellular glutamate increases activation of acid sensing ion channels, they lend their part to the destruction of the neuronal ionic environment [1]. Further after reperfusion there is an upsurge in production of superoxide, $\mathrm{NO}$ and peroxynitrate. Reperfusion-induced trau$\mathrm{ma}$ is actuated after the formation of these radicals in the vicinity of blood vessels. Matrix metallo proteases, highly potent proteases get activated as the level of free radical increases, which degrade collagen and laminins in the basal lamina. The degradation of these structural proteins disrupts the integrity of the vascular wall and BBB permeability [81]. Obviously energy-thirsty neurons fail to carry out their complex roles in maintaining posture, movement and sequencing language. Therefore, multimodal therapies that target the oxidative stress can greatly enhance therapeutic efficiency.

It is a well-known fact that cellular adhesion molecules (CAMs) have a significant contribution in the pathophysiology of ischemic stroke [27]. The up-regulation of CAMs is encountered within few hours of stroke and is responsible for the adhesion and migration of the leukocytes [62]. CAMs operate like anchors to the mobile leukocytes, which get adhered to the endothelial cells and then infiltrate into the injured brain. This permits an additional local ROS release by leukocytes and exacerbates ischemic injury [98]. Secondary damage originates as an aftermath of brain oedema, microvascular failure and vasomotor deficits leading to diminished blood perfusion and inflammation [28]. It requires activation of microglia and brain infiltration of peripheral inflammatory cells. These processes are especially detected during reperfusion resulting in an abrupt massive influx of ROS and leukocytes into the injured brain [7].

\section{Free radical sources in ischemic stroke The mitochondrial ROS production}

Mitochondria are central integrators and transducers of apoptotic signals in neurons. During the onset of ischemia, there is a marked drop in oxygen tension and glucose concentration in the brain tissue. This oxygenglucose deprivation (OGD) causes the accumulation of reducing intermediates and leads to an electron leak, which generates ROS [2]. ROS production depends on the metabolic state of mitochondria, for example, free 


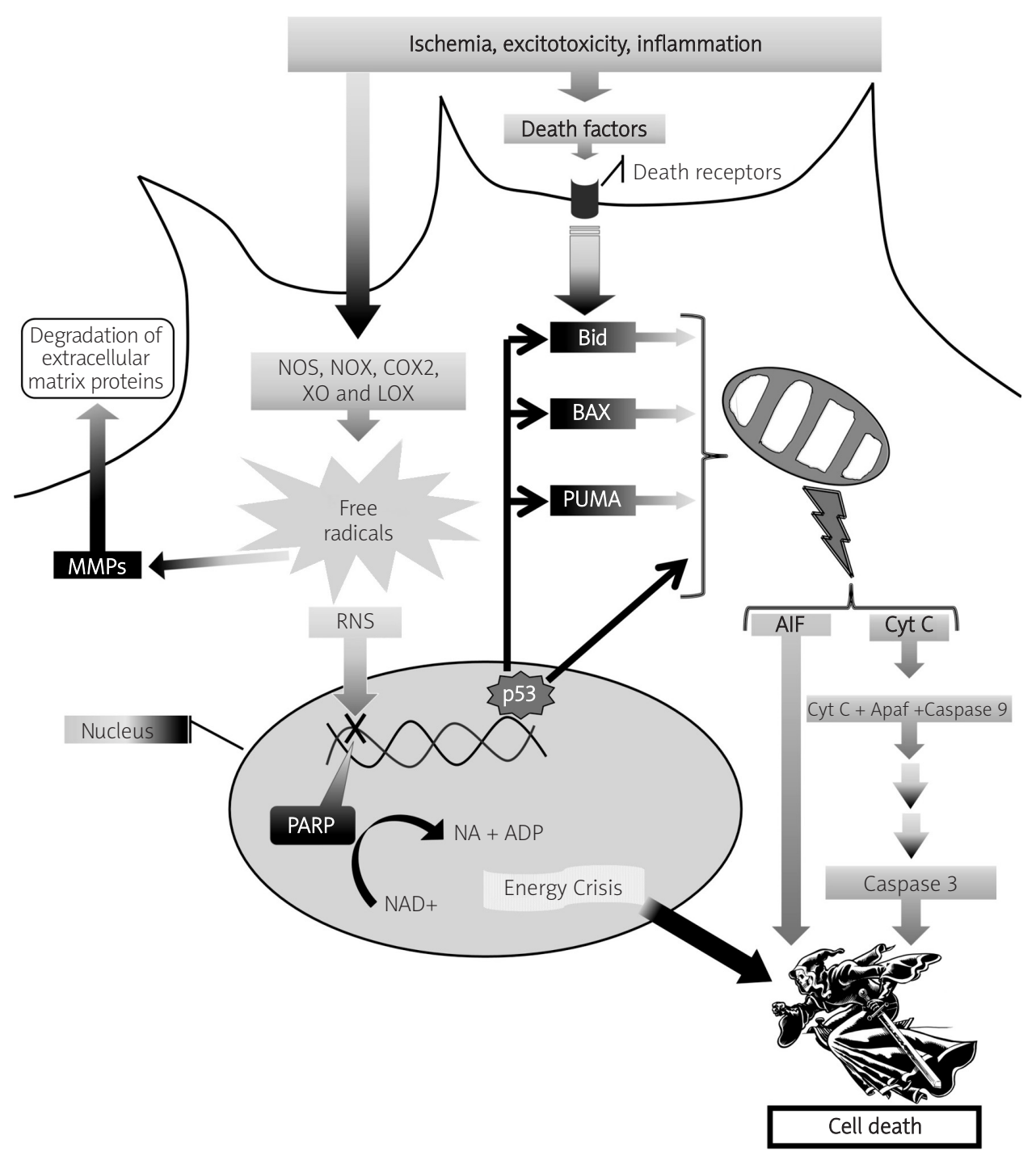

Fig. 2. Influence of oxidative stress on the neuronal cell death pathways during ischemic stroke.

radical production is greater during state IV (low electron flow and ATP synthesis, low ADP levels, high NADH/ $\mathrm{NAD}+$ ratio, and low oxygen consumption) respiration than in state III respiration [58]. In the absence of ADP, electrons derived from succinate can reversibly flow to complex 1 , generating increased $\mathrm{O}_{2}$ production, and for this reason, complex 1 is considered as the major physiologically and pathologically relevant ROS-generating site in the mitochondria [48].

Nitric oxide (NO•) is produced in mitochondria and is an important modulator of ROS production, as the ETC contains several $\mathrm{NO} \bullet$ reactive-redox metal centres. At physiological concentrations, $\mathrm{NO} \bullet$ modulates mitochondrial oxygen consumption by inhibiting cytochrome c oxidase in a reversible process [11,19]. A recent report suggested the involvement of p66Shc in mitochondrial ROS production. This protein forms a molecular complex with cytochrome c, subtracting electrons to catalyze the partial reduction of oxygen to form $\mathrm{O}_{2}$. P66Shc, partially localized in the inter-mitochondrial membrane space, is a downstream target of p53 and is indispensable for the increase in ROS production, cytochrome c release, dissipation of mitochondrial transmembrane potential and apoptosis [85]. ROS produced in the cell act in a positive feedback, leading to greater ROS production from mitochondria in a process termed ROS-induced ROS release. In mitochondriadependent apoptosis, molecular signalling returns to 
mitochondria, then triggers the release of critical apoptotic activators and effectors of cell death, such as cytochrome c or apoptosis-inducing factor, from the mitochondrial inter-membrane space [9].

\section{Xanthine oxidase as source of ROS}

Mounting evidence indicates that oxidative stress exacerbated during the phase of reperfusion is the main cause of brain injury in ischemia [41]. Ischemia damages the intricate mitochondrial balance, causing cessation in the oxidative phosphorylation and initiating the breakdown in the energetic equilibrium [88]. As a consequence of energy breakdown, levels of adenosine, inosine and hypoxanthine increase. Normally, hypoxanthine is converted by xanthine dehydrogenase to xanthine and uric acid. However, under ischemic conditions, xanthine dehydrogenase can transmute to xanthine oxidase $(\mathrm{XO})$, which uses oxygen as an electron acceptor [66]. XO generates significant amounts of superoxide anions, despite the low oxygen tension during the ischemia [6]. Under hypoxic conditions, XO metabolizes hypoxanthine and xanthine, to yield $\mathrm{O}_{2}^{-}$ and $\mathrm{H}_{2} \mathrm{O}_{2}$. Several investigations support the contribution of XO to tissue injury in ischemia/reperfusion. Oxypurinol, an inhibitor of XO was reported to block the production of ROS after ischemia [82]. These data confirm that oxidative stress originating from XO contributes to the neuronal injury during ischemia.

\section{NADPH oxidase}

NADPH oxidase (NOX) is a multi-subunit enzyme complex found in phagocytic leucocytes and vascular tissues. This membrane bound enzyme is suspected to be the key factor behind abnormal flaring up of the ROS level following ischemic reperfusion. Activated NADPH oxidase responds with increased generation of $\mathrm{O}_{2}^{-}$, which can react with water to generate hydroxyl radical and peroxynitrite by interacting with NO [56]. Among the five NOX isoforms (NOX 1-5), NOX2 and NOX4 isoforms are highly localized in the hippocampus CA1 and cerebral cortex. Experiments with NOX2 knockout animals and NOX2 inhibitor-treated animals showed a dramatic reduction in the infarct size, demonstrating the major role of NOX2 in oxidative stress-induced neuronal cell death following ischemia $[15,99]$. Hence, targeting specifically the vascular NOX4 or NOX2 isoforms might have advantages over non-specific suppression of the NADPH oxidase [68] In spite of many reports demonstrating the role of NOX, there is limited knowledge about the factors that govern neuronal NOX stimulation.

\section{NO synthases (NOS)}

Nitric oxide plays an important role in ROS mediated inflammation. Accumulating evidence indicates that NO can exert both protective and detrimental effects, depending upon the factors such as the chronological points after the onset of ischemic brain injury and isoform of the NOS and cellular source of NO $[31,47]$. Neuronal system harbours 3 kinds of NOS, endothelial NOS (eNOS), which maintains the cerebral blood flow, neuronal NOS (nNOS or NOS1) and inducible NOS (iNOS or NOS2) that can overproduce NO by distinct stimuli in glial cells [69]. Surprisingly, NO release from eNOS soon after brain ischemia is protective, by promoting vasodilation and by inhibiting microvascular aggregation and adhesion. However, after ischemia, NO produced by over-activation of nNOS and, later, NO release by de novo expression of iNOS contributes maximum to the brain damage. Another neurolytic path that involves NO is the production of highly reactive peroxynitrite, which can be regarded as a late neurotoxic event. The initial rise in NO after ischemia/reperfusion is a result of nNOS activation [42]. The mechanism of nNOS is governed by the intracellular calcium level. The nitric oxide production by eNOS is also calciumdependent, causes vasodilation and ameliorates blood flow in the regions of penumbra during ischemia. Hence, NO produced by eNOS is favourable for supporting collateral microcirculation [12]. Another isoform of NOS (iNOS) is one of the factors contributing to brain injury which will be upregulated in the NF-kB-dependent manner. Cytokine up-regulation after stroke is the key stimulus for the iNOS activation. The iNOS ensues in stretched nitric oxide production. Immediately after ischemia, the hypoxic condition flares up the level of iNOS [44]. iNOS has got a high activity several times greater than that of nNOS and hence, it has got a devastating consequence on the endothelium. The inhibition of iNOS shortens the leukocyte attachment to endothelium, and for that reason, it is one of the important targets against endothelial injury.

\section{Oxidative stress-mediated catastrophes \\ Inflammation}

The CNS responds to a variety of attacks with complex inflammatory processes. Inflammation takes 
part in the recovery after stroke and cerebral injury, but, simultaneously it is one of the threats which may worsen the stroke condition [35]. Inflammation during stroke is intermediated by both soluble factors, especially cytokines and also cellular components such as leukocytes and microglia, many of which have pro-inflammatory properties with promoting or destructive consequences $[3,89]$. ROS are generated as part of the natural immune response by inflammatory cells. ROS initiates the elevated expression of inflammatory mediators, such as adhesion molecules and cytokines immediately after stroke. These inflammatory factors establish a platform for leukocytes to adhere the vascular endothelial cells, thereby enhancing their accumulation. As a result, infiltration of leucocytes from the circulation into the ischemic regions shoots up within hours after the onset of stroke $[5,100]$. Leukocytes are factories of free radicals as they possess a multicomponent superoxide-generating system, NADPH oxidase. Superoxide generation by phagocytic oxidase takes place in phagosomes, whereas ROS by other oxidases are developed in the intracellular medium in smaller quantities [22]. Likewise, leucocytes (neutrophils) possess myeloperoxidase that generates highly reactive hypochloric acid, in the presence of $\mathrm{Cl}^{-}$and hydrogen peroxide. The effects of leukocytes on the pathogenesis of cerebral ischemia include initiation of thrombosis, decreased cerebral perfusion by occlusion and damage to the blood brain barrier (BBB) [76].

Drastic biochemical changes take place in the brain cells during the inflammatory response. Neurons are the most vulnerable to the ischemic attack among the cells in the brain. The glial cells (microglia and astrocytes) are important modulators of the immune response, which come to the fore after an ischemic attack. In the burst of respiratory activity, the activated microglia release significant amounts of ROS [59]. Free radical scavengers were found to significantly reduce the infarct volume in a stroke model in mice by limiting the microglial activation [91]. Microglial cells are the resident macrophages and serve as scavengers in the event of inflammation. The initial response is protective as the microglia clear foreign matter and debris, but following ischemia the continued response causes extensive cell damage. The microglia release cytotoxic substances following their activation, contributing to the ischemic damage [32,89].

COX-2 is another factor responsible for inflammationmediated damage after stroke. Among the brain cells, the COX-2 enzyme is predominantly expressed by dendritic profiles of glutamatergic neurons. Hyper activation and accumulation of COX-2 can be seen in the neurons, glia, vascular cells, and in inflammatory cells invading the brain during cerebral ischemia. Ischemic injury after cerebral artery occlusion can be attenuated by administering inhibitors of COX-2, with a comparatively wide therapeutic window (6 to 24 hours). In addition, ischemic trauma is aggravated in transgenic mice overexpressing COX-2 and is attenuated in COX-2 deficient mice [95]. This evidence demonstrates the involvement of COX-2 in the neuronal degeneration. However, destructive consequences of COX-2 are multifactorial and the mechanisms of the neuronal injury are not yet clear. The possible means of COX-2 lethality might be COX-2-mediated glutamate excitotoxicity initiating $\mathrm{Ca}^{2+}$ dysregulation, which in turn sets up the ischemic cascade. On the other hand, COX-2 contributes directly to the inflammatory reaction, thus heightening the deleterious effects to the ischemic brain [38].

\section{Neuronal cell death pathways}

ROS carry out a two-fold strategy of attack on the ischemic brain. The first mode of attack is the direct assault on proteins, lipids, nucleic acids and other macromolecular constituents of the cell. The second is the involvement of ROS in the cell signalling pathways leading to the apoptotic cell death after the ischemic insult. ROS act as a molecular trigger for ischemic cell death signalling $[65,81]$. The p53 signalling pathways are mediated by ROS released from the mitochondria. p53 is an important regulator of apoptosis following ischemic attack in the neurons. It is a transcription factor that controls the gene expression of products like Bax, Bid and p-53 upregulated modulator of apoptosis (PUMA), which act directly on the mitochondria and induce apoptosis [61]. There is a relationship between p53 signalling and oxidative stress via the upregulation of PUMA, a pro-apoptotic target. ROS are inextricably linked with mitochondrial dysfunction under hypoxic conditions. Mitochondrial dysfunction is implicated in both apoptotic and necrotic cell death by the initiation of cell signalling pathways. ROS trigger the release of the water-soluble protein cytochrome c from the mitochondrial membrane indirectly via the translocation of p53 [26]. The PUMA released by p53 interacts with the $\mathrm{Bcl}-2$ family of proteins to release cytochrome $\mathrm{c}$ from the mitochondria. SOD knockout mice show increased levels of cytochrome $c$ and extensive DNA damage after undergoing ischemic stroke, thus establishing the role 
of ROS as critical to apoptotic cell death $[30,46]$. Cytochrome $\mathrm{c}$ after several interactions activates caspase-9, which in turn initiates the cytochrome c-dependent caspase cascade. Caspases, or Cysteine-aspartic proteases, are associated with unwanted programmed cell death as part of the pathophysiology of several neurodegenerative diseases including ischemic stroke [79]. Caspase 9 activates caspase -3, 2, 6, 8 and 10. Caspases cleave many substrate proteins like poly (ADPribose) polymerase (PARP) and cause extensive damage to DNA [36].

Endothelial and neuronal cell apoptosis causes extensive neurological damage after ischemic stroke. It has been demonstrated that the apoptosis inhibitor TAT-BH4 protects the ischemic brain from injury by inducing neuroplasticity and preventing apoptosis in neuronal and endothelial cells [25]. Earlier studies have also shown that TAT-BH4 promotes endothelial cell survival by protecting from ROS-induced injury [30]. This highlights the role of ROS in endothelial cell signalling leading to apoptosis. Apoptosis in cells is determined by the delicate balance between pro-apoptotic and antiapoptotic factors. The Bcl-2 family and the caspase family are major players in the activation of the apoptotic signalling pathway. Caspase cascades are energy dependent and occur in the penumbral region which has a residual supply of blood. The release of apoptotic factors are induced by pro-apoptotic proteins like Bid, Bax and Bim [34]. These proteins disrupt mitochondrial membrane potential, triggering the release of cytochrome $\mathrm{c}$ through the permeability transition pore [64] (Fig. 2). Recent studies have discovered that the levels of the hormone adiponectin increase after ischemic stroke. Adiponectin is involved in the pro-apoptotic signalling cascade involving p38 mitogen-activated protein kinase (p38MAPK) and AMP-activated protein kinase (AMPK) [84].

\section{Excitotoxicity causes damage after ischemic stroke}

Oxidative stress, intracellular $\mathrm{Ca}^{2+}$ imbalance and glutamate excitotoxicity are closely interrelated in a complex series of biochemical cascades that occur after ischemic stroke [17]. Ca2+ overload have been established as a trigger for ischemic cell death in neurons [63]. The decrease of ATP generation following ischemia contributes to bio-energetic stress, which results in the inhibition of the $\mathrm{Na}^{+} / \mathrm{K}^{+}$ATPase and imbalances in $\mathrm{Na}^{+}$and $\mathrm{K}^{+}$levels in the neurons. As a con- sequence, there is an increase in intracellular $\mathrm{Na}^{+}$coupled with a significant decrease in the levels of $\mathrm{K}^{+}$in the cell. This leads to membrane depolarization or "anoxic depolarisation" of the neurons located in the core region of the ischemic lesion. Critical biochemical changes involved in the progression of the strokeinduced injury take place simultaneously with the onset of anoxic depolarisation. This results in the further deterioration and the expansion of the ischemic lesion [54].

Mitochondria act as cellular buffers of $\mathrm{Ca}^{2+}$ in the cell, accumulating the ions when the cytosolic levels exceed the set-point and releasing once the levels have been lowered by the action of membrane $\mathrm{Ca}^{2+}$ ATP-ases. The set-point is reached when the influx via the Calcium uniporter is balanced by the outflow from the mitochondrial $\mathrm{Na}^{+} / \mathrm{Ca}^{2+}$ exchanger [63]. The heightened levels of cytosolic $\mathrm{Ca}^{2+}$ puts a strain on the cell, resulting in the accumulation of $\mathrm{Ca}^{2+}$ above the maximum levels in the mitochondrial matrix [91]. This results in permeability transition, mitochondrial dysfunction, swelling and the release of cell death factors. Mitochondrial dysfunction produces more ROS resulting in a vicious cycle $[63,83]$. The accumulation of $\mathrm{Ca}^{2+}$ also generates more ROS during ischemia, augmenting the existing oxidative stress in the neurons. Investigations using gene knockout animals deficient in manganese superoxide dismutase, reported an increase in the levels of cytochrome $c$ after ischemia/reperfusion, leading to the conclusion that oxidative stress plays an important role in mitochondrial dysfunction $[30,45,75]$.

Ischemia is accompanied by the release of glutamate from synaptic vesicles and subsequent accumulation in the extracellular spaces (Fig. 1). The levels of extracellular glutamate have been found to increase after the onset of ischemia [52,53]. Glutamate is an important neurotransmitter in the mammalian CNS, performing vital roles in the development of neurons and synaptic plasticity $[24,60]$. There is evidence that the spontaneous release of glutamate from vesicles is mediated by the levels of cytosolic $\mathrm{Ca}^{2+}$. Experiments on the inhibition of the $\mathrm{N}$ type $\mathrm{Ca}^{2+}$ channel blocker were found to reduce glutamate levels significantly. The inhibition of the calcium uniporter was found to prevent glutamate excitotoxicity during ischemia. Another mechanism for the release of glutamate from neurons was found to be the reversal of the $\mathrm{Na}^{+} /$glutamate transporter. Free radicals are involved in the release of glutamate from neurons under ischemic conditions. Further, free radicals have been identified as agents that inhibit the uptake of glutamate by $\mathrm{Na}^{+} /$glutamate trans- 
porters resulting in excitotoxicity [50,54,94]. Antioxidant enzymes like SOD were found to block the accumulation of extracellular glutamate in cells under hypoxia.

Glutamate plays a central role in excitotoxic injury after stroke. Glutamate activates receptors linked to $\mathrm{Ca}^{2+}$ influx and affects mitochondrial function signi-ficantly. The influx of $\mathrm{Ca}^{2+}$ by the activation of glutamate mediated receptors triggers the uptake of Calcium by the mitochondria. The increase in the levels of $\mathrm{Ca}^{2+}$ in the mitochondrial matrix results in the events of membrane permeabilization and mitochondrial dysfunction discussed above [60]. Glutamate excitotoxicity results in a condition called extended neuronal depolarisation (END) [74]. The influx of $\mathrm{Ca}^{2+}$ has been identified as the cause of END through the activation of an injury induced calcium-permeable channel [51]. Experiments have led to the conclusion that glutamate neurotoxicity has two components: one dependent on the levels of $\mathrm{Na}$ and $\mathrm{K}$ ions and the other linked to the levels of extracellular $\mathrm{Ca}^{2+}$. Glutamate leads to the disruption of $\mathrm{Na}^{+}$levels and results in membrane depolarization and the secondary influx of water and $\mathrm{Cl}^{-}$. This is followed by the acute neuronal swelling contributing to excitotoxic injury. The osmotic gradients developed result in the influx of water into the cell, forming an oedema, which increases the intracranial pressure. The other component is the entry of $\mathrm{Ca}^{2+}$ via the activation of $\mathrm{N}$-methyl $\mathrm{D}$-aspartate glutamate mediated receptors. The complete loss of calcium homeostasis is the point beyond which neuronal damage cannot be reversed [17].

In vitro studies have shown that ischemia promotes glutamate-mediated free radical generation in hippocampal neurons. This increase in production of ROS coincides with the increase in intracellular calcium and membrane depolarization, reaching peak values at reoxygenation. The fall in ATP levels results in the inability to remove the $\mathrm{Ca}^{2+}$ accumulated in the cytosol via membrane ATPases. Thus, a direct relation exists between glutamate accumulations, rise in $\mathrm{Ca}^{2+}$ levels and free radical generation during hypoxia/reoxygenation [67]. In another mechanism of glutamate-induced injury, the high levels of extracellular glutamate interfere with the uptake of cystine through the cystine/glutamate antiporter. The decreased level of cystine in the cell leads to the depletion of intracellular glutathione (GSH). Glutathione acts as an antioxidant and the lack of it leads to severe oxidative damage from the action of ROS. This form of cell death is known as oxidative glutamate toxicity [71].

\section{Ischemic stroke therapy and the future}

The pathophysiology of stroke involves an array of complex and interconnected mechanisms, hence provides a range of potential molecular targets for the treatment of stroke. Modern stroke therapy is limited by a small therapeutic window which greatly diminishes the usefulness of the treatment. The nuclear factor erythroid 2-related factor 2 and antioxidant-response element (Nrf2-ARE) pathway is presently documented as the major cellular defence mechanism under oxidative stress. When activated, Nrf2 specifically targets genes bearing an antioxidant response element, which maintain redox homeostasis and influence the inflammatory response (heme oxygenase, 1-ferritin and glutathione peroxidise). A wide range of natural and synthetic small molecules are potent inducers of Nrf2 activity and has been shown to protect the brain against ischemic injury [49].

The role of oxidative stress and ROS in stroke injury has been firmly cemented by research and is one of the main concerns of stroke studies. The design of ROS scavengers and antioxidants, which can improve the antioxidant defences of the brain, has become the major part of modern stroke research. Randomized clinical studies involving antioxidants like vitamin E did not however provide conclusive results as to the reduction of ischemic stroke in patients [8]. This emphasises that the molecular mechanisms of ROS signalling after stroke are not fully understood. Since mitochondria are a major player in the ischemic cascade, antioxidants targeted at the organelle are being developed to reduce oxidative stress [78]. The use of recognised antioxidants like vitamin $\mathrm{E}$ and $\mathrm{C}$ as dietary supplements to reduce the risk of stroke is also being investigated [37]. The effects of multiple antioxidant supplementations are also being investigated [70]. Further, numerous studies have demonstrated a protective effect of hyperbaric oxygen (HBO) therapy in experimental ischemic brain injury. Tai et al. [80] have reported that HBO therapy effectively protects against heat-induced hypothalamic ischemia and oxidative damage, thermoregulatory dysfunction, and mortality against heatstroke. A heatstroke patient with multiple organ dysfunctions has also been successfully treated with HBO.

Moderate cerebral hypothermia initiated within 4 hour window after ischemic stroke has been found to reduce neuronal damage after ischemia by obstructing various inflammatory and injury-inducing pathways [20]. The major advantage of hypothermia treatment 
is the therapeutic window which is suited for clinical applications [72]. Further, the catalytic antioxidants which mimic endogenous antioxidants like superoxide dismutase and catalase can act as potent free radical scavengers and are expected to confer neuroprotection against oxidative stress [33].

\section{Conclusion}

Oxidative stress has been established as the major contributing factor in ischemic injury to the brain. In this article, we have discussed the role of oxidative stress at each stage of the ischemic injury. The biochemical cascades and signalling pathways triggered by oxidative stress have been highlighted keeping in view of the future development in stroke therapy, which is aimed at attenuating the effects of oxidative stress. The ROS induce neuronal damage via various biochemical pathways, offering numerous targets for stroke treatment. The complexity of mechanisms involved in the pathogenesis of stroke pose a great challenge to researchers studying the biochemistry of stroke. The development of novel antioxidant treatments is based on the complete understanding of the role of oxidative stress in stroke injury. Besides, the preference for antioxidant therapies on the oxidative process has to be evaluated carefully, since there are stages in the destructive neurochemical cascade that are regulated by oxidative mechanisms, and their inactivation may produce a reversed effect - potentiation or exacerbation of the processes leading to cell death. Moreover, stroke is a pathologically and etiologically heterogeneous disease and the risk factors may be different for the diverse stroke subtypes.

\section{References}

1. Aarts MM, Tymianski M. TRPMs and neuronal cell death. Pflugers Arch 2005; 451: 243-249.

2. Abramov AY, Scorziello A, Duchen MR. Three distinct mechanisms generate oxygen free radicals in neurons and contribute to cell death during anoxia and reoxygenation. J Neurosci 2007; 27: 11291138.

3. Ahmad M, Graham SH. Inflammation after Stroke: Mechanisms and Therapeutic Approaches. Transl Stroke Res 2010; 1: 74-84.

4. Altmeyer M, Hottiger MO. Poly (ADP-ribose) polymerase 1 at the crossroad of metabolic stress and inflammation in aging. Aging 2009; 1: 458-469.

5. Amantea D, Nappi G, Bernardi G, Bagetta G, Corasaniti MT. Postischemic brain damage: pathophysiology and role of inflammatory mediators. FEBS J 2009; 276: 13-26.

6. Abramov AY, Scorziello A, Duchen MR. Three Distinct Mechanisms Generate Oxygen Free Radicals in Neurons and Contribute to Cell
Death during Anoxia and Reoxygenation. The J Neurosci 2007; 27: 1129-1138.

7. Bașkaya MK, Dog`an A, Rao AM, Dempsey RJ. Neuroprotective effects of citicoline on brain edema and blood-brain barrier breakdown after traumatic brain injury. J Neurosurg 2000; 92: 448-452.

8. Behl C, Moosmann B. Oxidative nerve cell death in Alzheimer's disease and stroke: antioxidants as neuroprotective compounds. Biol Chem 2002; 383: 521-536.

9. Brandes RP. Triggering mitochondrial radical release: a new function for NADPH oxidases. Hypertension 2005; 45: 847-848.

10. Broderick JP, Brott T, Tomsick T, Miller R, Huster G. Intracerebral hemorrhage more than twice as common as subarachnoid hemorrhage. J Neurosurg 1993; 78: 188-191.

11. Brown GC, Cooper CE. Nanomolar concentrations of nitric oxide reversibly inhibit synaptosomal respiration by competing with oxygen at cytochrome oxidase. FEBS Lett 1994; 356: 295-298.

12. Bulhak AA, Jung C, Ostenson CG, Lundberg JO, Sjöquist PO, Pernow J. PPAR-alpha activation protects the type 2 diabetic myocardium against ischemia-reperfusion injury: involvement of the PI3-Kinase/Akt and NO pathway. Am J Physiol Heart Circ Physiol 2009; 296: 719-727.

13. Cantara S, Donnini S, Giachetti A, Thorpe PE, Ziche M. Exogenous TAT-BH4/Bcl-2 Peptide reverts coronary endothelial cell apoptosis induced by oxidative stress. J Vasc Res 2004; 41: 202-207.

14. Cao G, Xiao M, Sun F, Xiao X, Pei W, Li J, Graham SH, Simon RP, Chen J. Cloning of a novel Apaf-1-interacting protein: a potent suppressor of apoptosis and ischemic neuronal cell death. J Neurosci 2004; 24: 6189-6201.

15. Chen H, Song YS, Chan PH. Inhibition of NADPH oxidase is neuroprotective after ischemia-reperfusion. I Cereb Blood Flow Metab 2009; 29: 1262-1272.

16. Che Y, Wang JF, Shao L, Young T. Oxidative damage to RNA but not DNA in the hippocampus of patients with major mental illness. J Psychiatry Neurosci 2010; 35: 296-302.

17. Choi DW. Glutamate neurotoxicity in cortical cell culture is calcium dependent. Neurosci Letter 1985; 58: 293-297.

18. Choi DW. Calcium-mediated neurotoxicity: relationship to specific channel types and role in ischemic damage. Trends Neurosci 1988; 11: 465-469.

19. Cleeter MW, Cooper JM, Darley-Usmar VM, Moncada S, Schapira AH. Reversible inhibition of cytochrome c oxidase, the terminal enzyme of the mitochondrial respiratory chain, by nitric oxide. Implications for neurodegenerative diseases. FEBS Lett 1994; 345: 50-54.

20. Coimbra C, Wieloch T. Moderate hypothermia mitigates neuronal damage in the rat brain when initiated several hours following transient cerebral ischemia. Acta Neuropathol 1994; 87: 325-331.

21. Coyle J, Puttfarcken P. Oxidative stress, glutamate, and neurodegenerative disorders. Science 1993; 262: 689-695.

22. Cross AR, Segal AW. The NADPH oxidase of professional phagocytes - prototype of the NOX electron transport chain systems. Biochim Biophys Acta 2004; 1657: 1-22.

23. De Silva DA, Woon FP, Lee MP, Chen CL, Chang HM, Wong MC. Metabolic syndrome is associated with intracranial large artery disease among ethnic Chinese patients with stroke. J Stroke Cerebrovasc Dis 2009; 18: 424-427. 
24. Deshpande LS, Limbrick DD Jr, Sombati S, DeLorenzo RJ. Activation of a novel injury-induced calcium-permeable channel that plays a key role in causing extended neuronal depolarization and initiating neuronal death in excitotoxic neuronal injury. J Pharmacol Exp Ther 2007; 322: 443-452.

25. Donnini S, Solito R, Monti M, Balduini W, Carloni S, Cimino M, Bampton ET, Pinon LG, Nicotera P, Thorpe PE, Ziche M. Prevention of ischemic brain injury by treatment with the membrane penetrating apoptosis inhibitor, TAT-BH4. Cell Cycle 2009; 8: 1271-1278.

26. Endo H, Kamada H, Nito C, Nishi T, Chan PH. Mitochondrial translocation of p53 mediates release of cytochrome c and hippocampal CA1 neuronal death after transient global cerebral ischemia in rats. J Neurosci 2006; 26: 7974-7983.

27. Frijns CJ, Kappelle LJ. Inflammatory cell adhesion molecules in ischemic cerebrovascular disease. Stroke 2002; 33: 2115-2122.

28. Fritz HG, Bauer R. Secondary injuries in brain trauma: effects of hypothermia. J Neurosurg Anesthesiol 2004; 16: 43-52.

29. Fujimura M, Morita-Fujimura Y, Kawase M, Copin JC, Calagui B, Epstein CJ, Chan PH. Manganese superoxide dismutase mediates the early release of mitochondrial cytochrome $c$ and subsequent DNA fragmentation after permanent focal cerebral ischemia in mice. J Neurosci 1999; 19: 3414-3422.

30. Fujimura M, Morita-Fujimura Y, Noshita N, Sugawara T, Kawase M, Chan $\mathrm{PH}$. The cytosolic antioxidant copper/zinc-superoxide dismutase prevents the early release of mitochondrial cytochrome c in ischemic brain after transient focal cerebral ischemia in mice. J Neurosci 2000; 20: 2817-2824.

31. Gambaryan S, Kobsar A, Hartmann S, Birschmann I, Kuhlencordt PJ, Müller-Esterl W, Lohmann SM, Walter U. NO-synthase-/NOindependent regulation of human and murine platelet soluble guanylyl cyclase activity. J Thromb Haemost 2008; 6: 1376-1384.

32. Günther A, Küppers-Tiedt L, Schneider PM, Kunert I, Berrouschot J, Schneider D, Rossner S. Reduced infarct volume and differential effects on glial cell activation after hyperbaric oxygen treatment in rat permanent focal cerebral ischaemia. Eur J Neurosci 2005; 21: 3189-3194.

33. Golden TR, Patel M. Catalytic Antioxidants and Neurodegeneration. Antioxid Redox Sign 2009; 11: 555-570.

34. Gross A, Jockel J, Wei MC, Korsmeyer SJ. Enforced dimerization of BAX results in its translocation, mitochondrial dysfunction and apoptosis. EMBO J 1998; 17: 3878-3885.

35. Guo MF, Yu JZ, Ma CG. Mechanisms related to neuron injury and death in cerebral hypoxic ischaemia. Folia Neuropathol 2011; 49: 78-87.

36. Hakem R, Hakem A, Duncan GS, Henderson JT, Woo M, Soengas MS, Elia A, de la Pompa JL, Kagi D, Khoo W, Potter J, Yoshida R, Kaufman SA, Lowe SW, Penninger JM, Mak TW. Differential requirement for caspase 9 in apoptotic pathways in vivo. Cell 1998; 94: 339-3352.

37. Hirvonen T, Virtamo J, Korhonen P, Albanes D, Pietinen P. Intake of flavonoids, carotenoids, vitamins $\mathrm{C}$ and $\mathrm{E}$, and risk of stroke in male smokers. Stroke 2000; 31: 2301-2306.

38. Iadecola C, Gorelick PB. The Janus face of cyclooxygenase- 2 in ischemic stroke: shifting toward downstream targets. Stroke 2005; 36: 182-185.

39. Iadecola C, Yang G, Ebner TJ, Chen G. Local and propagated vascular responses evoked by focal synaptic activity in cerebellar cortex. J Neurophysiol 1997; 78: 651-659.
40. Ishibashi A, Yokokura Y. Outcomes in patients with ischemic cerebrovascular disease. Kurume Med J 1993; 78: 188-191.

41. Ishii T, Asai T, Urakami T, Oku N. Accumulation of macromolecules in brain parenchyma in acute phase of cerebral infarction/reperfusion. Brain Res 2010; 1321: 164-168.

42. Izci Y. Splenectomy may be a prophylactic treatment for cerebral ischemia? Med Hypotheses 2010; 75: 347-349.

43. Kahlert S, Zündorf G, Reiser G. Glutamate-mediated influx of extracellular $\mathrm{Ca}^{2+}$ is coupled with reactive oxygen species generation in cultured hippocampal neurons but not in astrocytes. J Neurosci Res 2005; 79: 262-271.

44. Keynes RG, Garthwaite J. Nitric oxide and its role in ischaemic brain injury. Curr Mol Med 2004; 4: 179-191.

45. Kim GW, Kondo T, Noshita N, Chan PH. Manganese superoxide dismutase deficiency exacerbates cerebral infarction after focal cerebral ischemia reperfusion in mice: implications for the production and role of superoxide radicals. Stroke 2002; 33: 809-815.

46. Kinouchi H, Epstein CJ, Mizui T, Carlson E, Chen SF, Chan PH. Attenuation of focal cerebral ischemic injury in transgenic mice overexpressing CuZn superoxide dismutase. Proc Natl Acad Sci 1991; 88: 11158-11162.

47. Korhonen R, Lahti A, Kankaanranta H, Moilanen E. Nitric oxide production and signaling in inflammation. Curr Drug Targets Inflamm Allergy 2005; 4: 471-479.

48. Kushnareva Y, Murphy AN, Andreyev A. Complex I-mediated reactive oxygen species generation: modulation by cytochrome $\mathrm{c}$ and NAD(P)+ oxidation-reduction state. Biochem J 2002; 368: 545-553.

49. Lakhan SE, Kirchgessner A, Hofer M. Inflammatory mechanisms in ischemic stroke: therapeutic approaches. J Transl Med 2009; 7: 97-108.

50. Lee J, Auyeung WW, Mattson MP. Interactive effects of excitotoxic injury and dietary restriction on microgliosis and neurogenesis in the hippocampus of adult mice. Neuromolecular Med 2003; 4: 179-196.

51. Limbrick DD Jr, Sombati S, DeLorenzo RJ. Calcium influx constitutes the ionic basis for the maintenance of glutamate-induced extended neuronal depolarization associated with hippocampal neuronal death. Cell Calcium 2003; 33: 69-81.

52. Li PA, Shuaib A, Miyashita H, He QP, Siesjö BK, Warner DS. Hyperglycemia enhances extracellular glutamate accumulation in rats subjected to forebrain ischemia. Stroke 2000; 31: 183-192.

53. Liu D, Gharavi R, Pitta M, Gleichmann M, Mattson MP. Nicotinamide prevents $N A D+$ depletion and protects neurons against excitotoxicity and cerebral ischemia: NAD+ consumption by SIRT1 may endanger energetically compromised neurons. Neuromolecular Med 2009; 11: 28-42.

54. Lipton P. Ischemic cell death in brain neurons. Physiol Rev 1999; 79: 1431-1568.

55. Lo EH, Dalkara T, Moskowitz MA. Mechanisms, challenges and opportunities in stroke. Nat Rev Neurosci 2003; 4: 399-415.

56. Loukogeorgakis SP, van den Berg MJ, Sofat R, Nitsch D, Charakida M, Haiyee B, de Groot E, MacAllister RJ, Kuijpers TW, Deanfield JE. Role of NADPH oxidase in endothelial ischemia/reperfusion injury in humans. Circulation 2010; 121: 2310-2316.

57. Lubos E, Handy DE, Loscalzo J. Role of oxidative stress and nitric oxide in atherothrombosis. Front Biosci 2008; 13: 5323-5344.

58. Madamanchi NR, Vendrov A, Runge MS. Oxidative stress and vascular disease. Arterioscler Thromb Vasc Biol 2005; 25: 29-38. 
59. Madinier A, Bertrand N, Mossiat C, Prigent-Tessier A, Beley A, Marie C, Garnier P. Microglial involvement in neuroplastic changes following focal brain ischemia in rats. PLoS One 2009; 4: e8101.

60. Mattson MP. Mitochondrial regulation of neuronal plasticity. Neurochem Res 2007; 32: 707-715.

61. Miyashita T, Reed JC. Tumor suppressor p53 is a direct transcriptional activator of the human bax gene. Cell 1995; 80: 293-299.

62. Mousa SA. Adhesion molecules: potential therapeutic and diagnostic implications. Methods Mol Biol 2010; 663: 261-276.

63. Nicholls DG. Mitochondrial calcium function and dysfunction in the central nervous system. Biochim Biophys Acta 2009; 1787: 1416-1424.

64. Oltvai ZN, Milliman CL, Korsmeyer SJ. Bcl-2 heterodimerizes in vivo with a conserved homolog, Bax, that accelerates programed cell death. Cell 1993; 74: 609-619.

65. Ozkul A, Akyol A, Yenisey C, Arpaci E, Kiylioglu N, Tataroglu C. Oxidative stress in acute ischemic stroke. J Clin Neurosci 2007; 14: 1062-1066.

66. Papi A, Contoli M, Gasparini P, Bristot L, Edwards MR, Chicca M, Leis M, Ciaccia A, Caramori G, Johnston SL, Pinamonti S. Role of xanthine oxidase activation and reduced glutathione depletion in rhinovirus induction of inflammation in respiratory epithelial cells. J Biol Chem 2008; 283: 28595-28606.

67. Perez Velazquez JL, Frantseva MV, Carlen PL. In vitro ischemia promotes glutamate-mediated free radical generation and intracellular calcium accumulation in hippocampal pyramidal neurons. J Neurosci 1997; 17: 9085-9094

68. Raz L, Zhang QG, Zhou CF, Han D, Gulati P, Yang LC, Yang F, Wang RM, Brann DW. Role of Rac1 GTPase in NADPH oxidase activation and cognitive impairment following cerebral ischemia in the rat. PLoS One 2010; 5: e12606.

69. Rosa SC, Judas F, Lopes MC, Mendes AF. Nitric oxide synthase isoforms and NF-kappaB activity in normal and osteoarthritic human chondrocytes: regulation by inducible nitric oxide. Nitric Oxide 2008; 19: 276-283.

70. Sánchez-Moreno C, Jiménez-Escrig A, Martín A. Stroke: roles of B vitamins, homocysteine and antioxidants. Nutr Res Rev 2009; 22: 49-67

71. Schubert D, Piasecki D. Oxidative glutamate toxicity can be a component of the excitotoxicity cascade. J Neurosci 2001; 21: $7455-$ 7462 .

72. Shintani Y, Terao Y, Ohta H. Molecular mechanisms underlying hypothermia-induced neuroprotection. Stroke Res Treat 2010; 2011: 809874.

73. Siesjo BK. Historical overview. Calcium, ischemia, and death of brain cells. Ann NY Acad Sci 1988; 522: 638-661.

74. Sombati S, Coulter DA, DeLorenzo RJ. Neurotoxic activation of glutamate receptors induces an extended neuronal depolarization in cultured hippocampal neurons. Brain Res 1991; 566: 316-319.

75. Soane L, Kahraman S, Kristian T, Fiskum G. Mechanisms of impaired mitochondrial energy metabolism in acute and chronic neurodegenerative disorders. J Neurosci Res 2007; 85: 3407-3415.

76. Stocker R, Keaney JF. Role of oxidative modifications in atherosclerosis. Physiol Rev 2004; 84: 1381-478.

77. Stoll G, Bendszu M. Inflammation and atherosclerosis: novel insights into plaque formation and destabilization. Stroke 2006; 37: 1923-1932
78. Subramanian S, Kalyanaraman B, Migrino RQ. Mitochondrially targeted antioxidants for the treatment of cardiovascular diseases. Recent Pat Cardiovasc Drug Discov 2010; 5: 54-65.

79. Sugawara T, Noshita N, Lewén A, Gasche Y, Ferrand-Drake M, Fujimura M, Morita-Fujimura Y, Chan PH. Overexpression of copper/zinc superoxide dismutase in transgenic rats protects vulnerable neurons against ischemic damage by blocking the mitochondrial pathway of caspase activation. J Neurosci 2002; 22: 209-217.

80. Tai PA, Chang CK, Niu KC, Lin MT, Chiu WT, Lin JW. Reduction of ischemic and oxidative damage to the hypothalamus by hyperbaric oxygen in heatstroke mice. J Biomed Biotechnol 2010; 2010: 609526.

81. Taylor JM, Crack PJ. Impact of oxidative stress on neuronal survival. Clin Exp Pharmacol Physiol 2004; 31: 397-306.

82. Terzi C, Kuzu A, A lar AK, Kale IT, Tanik A, Köksoy C. Prevention of deleterious effects of reperfusion injury using one-week highdose allopurinol. Dig Dis Sci 2001; 46: 430-437.

83. Thayer SA, Miller RJ. Regulation of the intracellular free calcium concentration in single rat dorsal root ganglion neurones in vitro. J Physiol 1990; 425: 85-115.

84. Thundyil J, Tang SC, Okun E, Shah K, Karamyan VT, Li YI, Woodruff TM, Taylor SM, Jo DG, Mattson MP, Arumugam TV. Evidence that adiponectin receptor 1 activation exacerbates ischemic neuronal death. Exp Transl Stroke Med 2010; 2: 15.

85. Trinei M, Giorgio M, Cicalese A, Barozzi S, Ventura A, Migliaccio E, Milia E, Padura IM, Raker VA, Maccarana M, Petronilli V, Minucci S, Bernardi P, Lanfrancone L, Pelicci PG. A p53-p66Shc signalling pathway controls intracellular redox status, levels of oxidation-damaged DNA and oxidative stress-induced apoptosis. Oncogene 2002; 21: 3872-3878.

86. Tuladhar AM, de Leeuw FE. Poststroke dementia - what's in a name? Nat Rev Neurol 2010; 6: 63-64.

87. Valko M, Leibfritz D, Moncol J, Cronin MT, Mazur M, Telser J. Free radicals and antioxidants in normal physiological functions and human disease. Int J Biochem Cell Biol 2007; 39: 44-84.

88. Vanlangenakker N, Vanden Berghe T, Krysko DV, Festjens N, Vandenabeele P. Molecular mechanisms and pathophysiology of necrotic cell death. Curr Mol Med 2008; 8: 207-220.

89. Wang H, Hitron IM, ladecola C, Pickel VM. Synaptic and vascular associations of neurons containing cyclooxygenase- 2 and nitric oxide synthase in rat somatosensory cortex. Cereb Cortex 2005; 15: $1250-1260$.

90. Wang J, Doré S. Heme oxygenase-1 exacerbates early brain injury after intracerebral haemorrhage. Brain 2007; 130: 1643-1652.

91. Wang J, Tsirka SE. Neuroprotection by inhibition of matrix metalloproteinases in a mouse model of intracerebral haemorrhage. Brain 2005; 128: 1622-1633.

92. Wang X, Mori T, Sumii T, Lo EH. Hemoglobin-induced cytotoxicity in rat cerebral cortical neurons: caspase activation and oxidative stress. Stroke 2002; 33: 1882-1888.

93. Weinberger J. Diagnosis and prevention of atherosclerotic cerebral infarction. CNS Spectr 2005; 10: 553-564.

94. Won SJ, Kim DY, Gwag BJ. Cellular and molecular pathways of ischemic neuronal death. J Biochem Mol Biol 2002; 35: 67-86.

95. Xiang Z, Thomas S, Pasinetti G. Increased neuronal injury in transgenic mice with neuronal overexpression of human cyclooxy- 
genase- 2 is reversed by hypothermia and rofecoxib treatment. Curr Neurovasc Res 2007; 4: 274-279.

96. Xiong ZG, Chu XP, Simon RP. Acid sensing ion channels - novel therapeutic targets for ischemic brain injury. Front Biosci 2007; 12: $1376-1386$

97. Yager JY, Kala G, Hertz L, Juurlink BH. Correlation between content of high-energy phosphates and hypoxic-ischemic damage in immature and mature astrocytes. Brain Res Dev Brain Res 1994; 82: 62-68.

98. Yilmaz G, Granger DN. Cell adhesion molecules and ischemic stroke. Neurol Res 2008; 30: 783-793.

99. Zhang QG, Raz L, Wang R, Han D, De Sevilla L. Estrogen attenuates ischemic oxidative damage via an estrogen receptor alphamediated inhibition of NADPH oxidase activation. J Neurosci 2009; 29: 13823-13836.

100. Zheng Z, Yenari MA. Post-ischemic inflammation: molecular mechanisms and therapeutic implications. Neurol Res 2004; 26 : 884-892. 